\title{
Efficacy and Safety of Preservative-free Latanoprost Eyedrops Compared with Preserved Prostaglandin Analogues in Patients with Open-angle Glaucoma
}

\author{
Hyo Jin Seong, ${ }^{1,2}$, Kwanghyun Lee ${ }^{1,2}$, Seung Jae Lee ${ }^{1,2}$, Sangah Kim ${ }^{3}$, Jong Woon Park ${ }^{2}$ \\ ${ }^{1}$ Department of Ophthalmology, Yonsei University College of Medicine, Seoul, Korea \\ ${ }^{2}$ Department of Ophthalmology, National Health Insurance Service Ilsan Hospital, Goyang, Korea \\ ${ }^{3}$ Siloam Eye Hospital, Seoul, Korea
}

Purpose: To evaluate the safety and efficacy of preservative-free (PF) latanoprost in glaucoma patients.

Methods: In this prospective, open-label, observational study, a total of 27 primary open-angle glaucoma patients who used benzalkonium chloride-preserved prostaglandin analogues for at least 6 months were enrolled. After changing the eye drops to PF lataprost, the intraocular pressure (IOP) and ocular surface symptoms and signs were evaluated in all patients on days 0 (first visit, D0), 45 (D45), and 90 (D90).

Results: Mean IOP remained stable during the study period $(14.0 \pm 2.4 \mathrm{mmHg}$ at D0, $13.9 \pm 2.0 \mathrm{mmHg}$ at D45, $13.7 \pm 2.2$ $\mathrm{mmHg}$ at D90; $p=0.603$ ). Mean deviation, pattern standard deviation, and best-corrected visual acuity were similar before and after eye drops replacement. Bulbar conjunctival hyperemia, corneal staining, and conjunctival staining were significantly decreased over 90 days ( $p=0.025, p<0.001, p=0.020$, respectively). The ocular surface disease index score showed a statistically significant improvement from $26.4 \pm 18.5$ at D0 to $19.8 \pm 17.0$ at D45 and $15.7 \pm 15.6$ at D90 ( $p<0.001)$. In the evaluation of ocular tolerability, burning symptoms and dryness were significantly decreased ( $p=0.001, p=0.040$ ).

Conclusions: The effects of PF latanoprost on reducing IOP were comparable with those of benzalkonium chloride-preserved prostaglandin analogues, but side effects on the ocular surface were much less pronounced when PF latanoprost was used. With this efficacy, PF latanoprost could slow the progression of glaucoma by increasing patient compliance.

Key Words: Glaucoma, Pharmaceutical preservatives, Synthetic prostaglandins

As one of major blindness diseases, glaucoma is a progressive, chronic, and vision-threatening disease in which retinal ganglion cells and axons are damaged. There are

Received: January 12, 2021 Final revision: April 5, 2021

Accepted: April 30, 2021

Corresponding Author: Jong Woon Park, MD, PhD. Department of Ophthalmology, National Health Insurance Service Ilsan Hospital, 100 Ilsan-ro, Ilsandong-gu, Goyang 10444, Korea. Tel: 82-31-900-0964, Fax: 82-31-900-0049, E-mail: 2eye2@nhimc.or.kr various risk factors for glaucoma, but intraocular pressure (IOP) is the most important among them. As the elderly population increases and diagnostic technology advances, the prevalence of glaucoma also increases especially in the 40 to 80 years old group. The global prevalence of glaucoma in 40 to 80 years old group is $3.54 \%$, and for primary open angle glaucoma (POAG), the worldwide prevalence was 3.05\% [1]. In South Korea, the prevalence of POAG is varied from $2.6 \%$ to $4.7 \%$ depending on the study design [2-4]. 
Topical glaucoma medications are the first-line treatment to decrease IOP to prevent further glaucoma progression. Among many topical medications, prostaglandin analog eye drops (PGAs) are often prescribed as first-line IOP-reducing agents for open-angle glaucoma [5]. PGAs increase the uveal-scleral outflow of aqueous humor by binding to prostaglandin $\mathrm{F} 2 \alpha$ receptor (FP receptor). PGAs are known to be the most effective agents for open-angle glaucoma, as they can reduce IOP by $25 \%$ to $35 \%$. However, PGAs and other eye drops currently in the market contain preservatives and phosphate buffers that help to prevent them from infection and denaturation. Benzalkonium chloride (BAK) is the most used preservative because of its antimicrobial activity, which acts by disrupting cell membranes and potentiating cell death. However, BAK also has a high affinity for human cell membrane proteins and induces inflammatory and pro-apoptotic changes in ocular tissues, inducing cellular toxicity in a dose- and time-dependent manner [6-8]. Therefore, long-term use of BAK-preserved PGAs can cause dry eye syndrome, meibomian gland dysfunction, conjunctivitis, or corneal toxicity [9].

Therefore, this study aimed to evaluate the efficacy and safety of preservative-free (PF) latanoprost on POAG patients who previously used BAK-preserved PGAs.

\section{Materials and Methods}

This prospective, open-label, observational study followed the tenets of the Declaration of Helsinki. This study was approved by the institutional review board of the $\mathrm{Na}$ tional Health Insurance Service Ilsan Hospital (NHIMC 2019-09- 001-002). Written informed consent was obtained from all participants before any study-related procedure.

From November 25, 2019, to November 24, 2020, POAG patients aged between 19 and 80 years who visited the secondary level eye care clinic were enrolled in the study. POAG was defined as a glaucoma with open anterior chamber angle with following characteristic features: typical glaucomatous change of optic disc, retinal nerve fiber layer defect, and corresponding glaucomatous visual field (VF) defect. Also, there should be no secondary cause of optic neuropathy. Glaucomatous VF defect was defined as 1) a cluster of 3 or more points with a probability $<5 \%$ on the pattern deviation map in at least one hemifield, including at least one point with a probability of $<1 \%$, or 2 ) a pat- tern standard deviation with a probability of $<5 \%$, or 3 ) glaucoma hemifield test values outside the normal limits.

Inclusion criteria were 1) IOPs that remain stable below $21 \mathrm{mmHg}$ with PGA use for 6 months, 2) a stable VF based on at least two reliable VF test performed within the last 6 months, 3) central corneal thickness within the range 500 to $580 \mu \mathrm{m}, 4)$ best-corrected visual acuity (BCVA) in both eyes is 20 / 80 or higher based on the Snellen vision chart, and 5) good drug compliance as defined as following at least $80 \%$ of the guidelines for use and frequency of eye drops.

Exclusion criteria were 1) previous history of primary angle-closure glaucoma, congenital glaucoma, secondary glaucoma due to trauma, inflammation, tumor, dense cataract, or drugs such as steroids, 2) previous history of acute angle-closure glaucoma, 3) BCVA in both eyes of less than 20 / 80 based on the Snellen vision chart, 4) clinical evidence of inflammation (in case of chronic intraocular inflammation or a history of intraocular inflammation within 3 months immediately prior to participation in the study), 5) use of contact lens, 6) abnormal corneal condition that could make it difficult to measure IOP, 7) central corneal thickness outside the 500 to $580 \mu \mathrm{m}$ range, 8) history of surgery that affects dryness, such as refractive surgery and cataract surgery within the last 3 months, 9) history of punctal occlusion surgery or surgical plan during the clinical study, 10) hypersensitivity to PGAs such as allergic contact dermatitis, 11) use of any eyedrops for dry eye symptoms other than carboxymethyl cellulose during the study period or 12) conditions of the optic disc or visual field judged by the investigator JWP that PGAs alone is not sufficient (i.e., advanced cupping or significant deterioration of VF indices within 6 months).

At the baseline visit (D0), patients included in the study switched their medications from BAK-preserved PGAs to PF latanoprost (Xalost-S; Taejoon Pharmaceutical, Seoul, Korea). All subjects were instructed to administer one drop of PF latanoprost to the glaucoma eye once every evening. All patients instilled the eyedrop for 90 days and visited our clinic on the 45th and 90th day after participation (D45 and D90). At each visit (D0, D45, and D90), BCVA, IOP, conjunctival hyperemia, corneal and conjunctival staining, and tear break-up time (TBUT) were evaluated. All ophthalmic examinations were performed by two examiners, KL and SK, glaucoma specialists with considerable experience. In the case of disagreement between the 
two examiners' evaluations, JWP made the final decision. A questionnaire on the ocular surface disease index (OSDI) and ocular tolerability was administered at each visit. The VF test was performed on D0 and D90.

IOP was measured twice at each visit using a Goldmann applanation tonometer and the mean value was used for analysis. To reduce diurnal variation of IOP, patients were instructed to visit the hospital at the same time as their first visit. Conjunctival hyperemia was evaluated on a scale of 0 to 4 based on the Efron grading scale [10]. For corneal staining, the Oxford schema grading system was used under cobalt blue light after the instillation of fluorescein and scored from 0 to 3 . Conjunctival staining was evaluated according to the National Eye Institute (NEI scale). After fluorescein staining, we divided the conjunctiva into six equal parts and graded each area by assigning a score from 0 to 3 [11]. The TBUT was measured by instilling a Fluorescein paper strip (FLUO 900; Haag-Streit AG, Koeniz, Switzerland) on the conjunctival sac, blinking for several seconds, and then measuring the time until the first defect of the fluorescent dye appeared in the dyed tear film layer under a cobalt blue light source [12].

The OSDI was composed of a total of 12 questions and the score given was directly proportional to the severity of the symptoms. OSDI was evaluated on a scale from 0 to 100 and classified as normal (0-12), minimal (13-22), moderate (23-32), and severe (33-100) [7]. For ocular tolerability evaluation, the severity of symptoms was evaluated by scoring from 0 to 3 for the following 8 symptoms: burning, stickiness, itching, sandiness, dryness, blurred vision, light sensitivity, and pain.

VF test was measured by the C24-2 Swedish Interactive Thresholding Algorithm standard program using Humphrey Field Analyzer (Carl-Zeiss Meditec, Dublin, CA, USA). The VF test was considered appropriate when the fixation loss was less than $20 \%$ and the false positive and false negative rates were less than 33\%.

Data were analyzed using R software ver. 3.6.3 (R Foundation for Statistical Computing, Vienna, Austria). Results are presented as mean \pm standard deviation, numbers, and percentages. Statistical analysis was performed using the one-way random effects analysis of variance, followed by a pairwise Tukey's post hoc test. A p-value of less than 0.05 was considered statistically significant.

\section{Results}

\section{Baseline characteristics}

Of the 30 patients included in this study, three were eliminated, two changed their mind and withdrew from the study, and one was eliminated owing to the addition of a different eye drop to treat dry eye disease. Before changing the eye drops, 16 patients (59.3\%) were using bimatoprost (Lumigan; Allergan, Irvine, CA, USA), nine patients $(33.3 \%)$ were using latanoprost (Xalatan; Pfizer, New York, NY, USA), and two patients (7.4\%) were using tafluprost (Taflotan; Santen Pharmaceutical, Osaka, Japan). Sixteen $(59.3 \%)$ were men with a mean age of $62.7 \pm 7.7$ years. Mean BCVA was $0.8 \pm 0.2$, and IOP was $14.0 \pm 2.4 \mathrm{mmHg}$. The mean deviation of the Humphrey test was $-4.7 \pm 5.2$ $\mathrm{dB}$, and the pattern standard deviation was $5.3 \pm 4.3 \mathrm{~dB}$ (Table 1).

\section{Objective assessments}

Mean BCVA and IOP did not change significantly over 90 days after switching to PF latanoprost. Limbal conjunctival hyperemia was not significantly different from baseline, but bulbar conjunctival hyperemia significantly decreased after 90 days. Corneal/conjunctival disease was also significantly decreased. TBUT at D90 was not significantly different from TBUT (Table 2).

\section{Subjective assessments}

After changing to PF latanoprost, the OSDI score showed a statistically significant decrease. In the evaluation of ocular tolerance, burning symptoms, and dryness

Table 1. Clinical and demographic data of the patients $(n=27)$

\begin{tabular}{lc}
\hline Characteristics & Value \\
\hline Sex (male) & $16(59.3)$ \\
Age (yr) & $62.7 \pm 7.7(42.0$ to 74.0$)$ \\
Best-corrected visual acuity & $0.8 \pm 0.2(0.3$ to 1.0$)$ \\
Intraocular pressure (mmHg) & $14.0 \pm 2.4(8.0$ to 19.0$)$ \\
Mean deviation (dB) & $-4.7 \pm 5.2(-22.3$ to 1.64$)$ \\
Pattern standard deviation $(\mathrm{dB})$ & $5.3 \pm 4.3(0.91$ to 15.54$)$ \\
\hline
\end{tabular}

Values are presented as number (\%) or mean \pm standard deviation (range). 
Table 2. Changes in BCVA, IOP, ocular surface parameters, TBUT, and visual field score after treatment with preservative-free latanoprost $(\mathrm{n}=27)$

\begin{tabular}{lrrrr}
\hline Characteristics & Baseline & 45 day & 90 day & $p_{\text {-value }}^{*}$ \\
\hline BCVA & $0.8 \pm 0.2$ & $0.8 \pm 0.2$ & $0.8 \pm 0.2$ & 0.342 \\
IOP (mmHg) & $14.0 \pm 2.4$ & $13.9 \pm 2.0$ & $13.7 \pm 2.2$ & 0.603 \\
Bulbar injection & $1.3 \pm 0.9$ & $1.2 \pm 0.7$ & $1.0 \pm 0.7$ & 0.025 \\
Limbal injection & $1.2 \pm 0.8$ & $1.1 \pm 0.7$ & $1.0 \pm 0.7$ & 0.052 \\
Cornea staining & $1.6 \pm 1.0$ & $1.0 \pm 0.8$ & $0.8 \pm 0.9$ & $<0.001$ \\
Conjunctiva staining & $5.9 \pm 3.4$ & $5.6 \pm 2.5$ & $4.6 \pm 3.4$ & 0.020 \\
TBUT (sec) & $7.4 \pm 3.5$ & $7.4 \pm 3.1$ & $6.9 \pm 2.6$ & 0.369 \\
MD (dB) & $-4.7 \pm 5.2$ & NA & $-5.1 \pm 5.7$ & 0.398 \\
PSD (dB) & $5.3 \pm 4.3$ & NA & $5.4 \pm 4.4$ & 0.869 \\
\hline
\end{tabular}

Values are presented as the mean \pm standard deviation.

$\mathrm{BCVA}=$ best-corrected visual acuity; IOP = intraocular pressure; TBUT = tear break-up time; $\mathrm{MD}=$ mean deviation; $\mathrm{NA}=$ not applicable; PSD = pattern standard deviation.

* One-way random effects analysis of variance statistical significance: $p<0.05$ indicates statistical significance.

Table 3. Changes in OSDI and ocular tolerability score after treatment with preservative-free latanoprost $(n=27)$

\begin{tabular}{lcccc}
\hline Characteristics & Baseline & 45 day & 90 day & $p_{\text {-value }}^{*}$ \\
\hline OSDI & $26.4 \pm 18.5$ & $19.8 \pm 17.0$ & $15.7 \pm 15.6$ & \\
Tolerability & & & & \\
$\quad$ Burning sensation & $0.9 \pm 0.8$ & $0.3 \pm 0.6$ & $0.3 \pm 0.6$ & 0.001 \\
Sticky sensation & $0.3 \pm 0.4$ & $0.3 \pm 0.6$ & $0.1 \pm 0.6$ & 0.615 \\
Itching sensation & $0.6 \pm 0.8$ & $0.2 \pm 0.4$ & $0.4 \pm 0.8$ & 0.102 \\
Blurred vision & $0.7 \pm 0.9$ & $0.4 \pm 0.7$ & $0.5 \pm 0.9$ & 0.193 \\
Sandiness & $0.7 \pm 0.8$ & $0.4 \pm 0.6$ & $0.5 \pm 0.8$ & 0.398 \\
Dryness & $1.1 \pm 1.1$ & $0.6 \pm 0.9$ & $0.5 \pm 0.9$ & 0.040 \\
Light sensitivity & $0.8 \pm 1.0$ & $0.8 \pm 1.0$ & $0.6 \pm 1.0$ & 0.627 \\
Pain & $0.6 \pm 0.7$ & $0.2 \pm 0.5$ & $0.3 \pm 0.8$ & 0.085 \\
\hline
\end{tabular}

Values are presented as the mean \pm standard deviation.

OSDI = ocular surface disease index questionnaire.

*One-way random effects analysis of variance statistical significance: $p<0.05$ indicates statistical significance.

were significantly decreased. On the other hand, there were no significant differences in stickiness, itchiness, blurred vision, foreign body sensation, light sensitivity, and pain, according to the presence or absence of preservatives (Table 3 ).

\section{Post hoc analysis}

We performed the post hoc tests for six parameters that showed significant differences before and 90 days after the change to PF latanoprost. Corneal staining, burning, and
OSDI score decreased statistically significantly at day 45 after the change. On the other hand, there were no statistically significant differences in conjunctival hyperemia, conjunctival staining, and dryness at D45 (Fig. 1).

\section{Discussion}

The purpose of this study was to demonstrate the stability and efficacy of PF latanoprost as a treatment for glaucoma. With the switch to PF latanoprost, IOP remained 
A
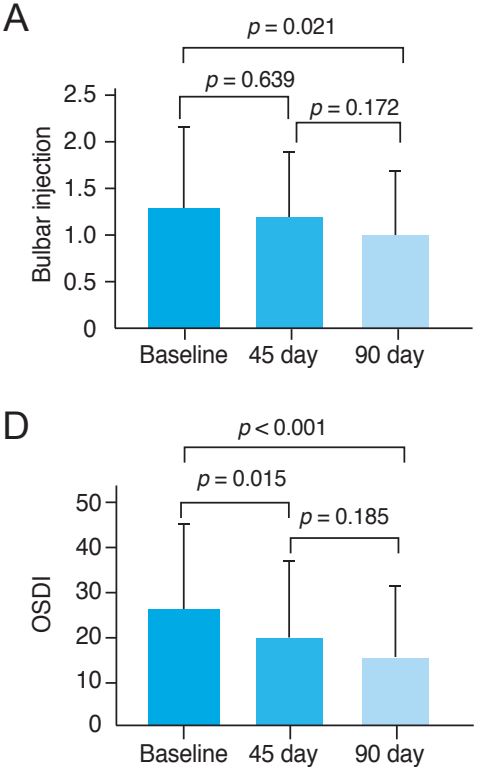

B

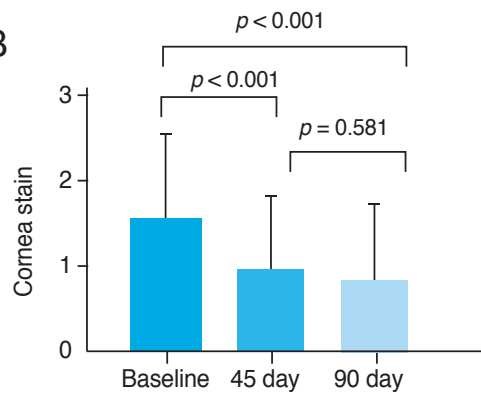

$E$

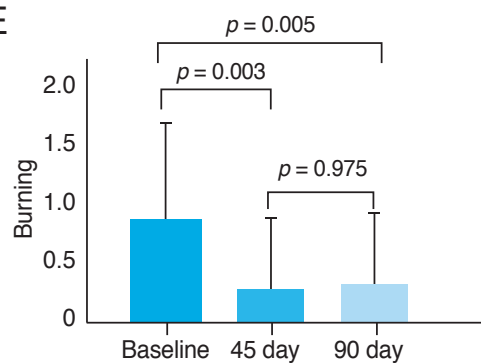

C

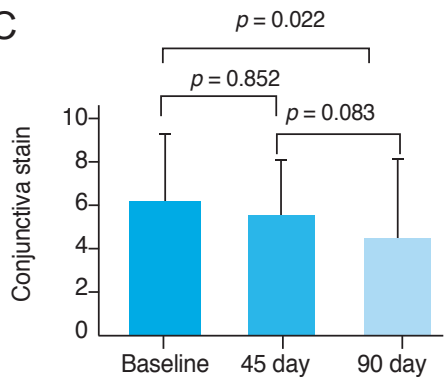

$\mathrm{F}$

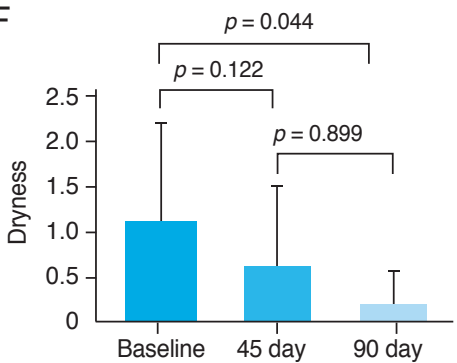

Fig. 1. Results of the post-hoc analysis. Six parameters (A, bulbar injection; B, cornea stain; C, conjunctiva stain; D, ocular surface disease index [OSDI]; E, burning; F, dryness) showed significant differences 90 days after administration of the preservative-free latanoprost. Corneal staining, burning, and OSDI score were significantly improved 45 days after administration $(p<0.001, p=0.003, p=0.015)$. On the other hand, there were no statistically significant differences in conjunctival hyperemia, conjunctival staining, and dryness 45 days after administration $(p=0.639, p=0.852, p=0.122)$. $p$-value: one-way random effects analysis of variance with Tukey post hoc.

stable and ocular discomfort was significantly reduced.

The primary treatment for glaucoma is lowering IOP through anti-glaucoma eye drops. PGAs have the strongest IOP-lowering effect among anti-glaucoma eye drops, and patients are highly compliant as these drops need to be used only once daily [9]. PGAs include latanoprost, bimatoprost, travoprost, and tafluprost, and they work by increasing the outflow of aqueous fluid from the anterior chamber. However, ocular surface tissues may be adversely affected by preservatives included in commercially available PGAs to inhibit the proliferation of microorganisms.

BAK, a representative preservative, is a weak base and has superior antibacterial properties by destroying the cell membrane of microorganisms more efficiently than other preservatives $[13,14]$. Owing to these properties, it has been commonly used in various eye drops. However, since it also has a high affinity for membrane proteins, long-term use may cause various ocular surface symptoms through chronic inflammation, inhibition of proliferation, and apoptosis of conjunctival and corneal epithelial cells. As a result, the patient's quality of life and drug compliance may decrease, and surgical outcomes, such as cataract sur- gery or trabeculectomy, may be affected [15-17].

Therefore, there have been efforts to develop PF anti-glaucoma eye drops. In addition, studies comparing the effects of PF eye drops and conventional eye drops have been conducted in various countries [8,16,18-22]. In most studies, the effects of both eye-drops on IOP were similar, but $\mathrm{PF}$ eye drops tended to be more effective in reducing ocular surface symptoms and signs. In our study, IOP remained stable during the study period. Similar results have been reported by other researchers, except Garcia-Feijoo et al. [18], who reported that BAK-free travoprost was effective in lowering IOP compared to preserved bimatoprost or latanoprost.

Bulbar conjunctival hyperemia and corneal/conjunctival were found to improve by using PF eye drops in our study as well as in other studies. Our research team and Misiuk-Hojlo et al. [16] reported no improvement in tear breakdown time, while others reported significantly improved results $[8,20,22]$. The OSDI was significantly decreased in our study, and a previous study [20] presented similar results. The patients' subjective assessments of tolerability improved in our study as well as in other studies $[8,16,20$ 22]. In general, redness, burning/stinging, and foreign 
body sensation were significantly improved.

There are strengths and limitations to this study. This was a prospective study conducted on POAG patients, one of the common types of glaucoma in South Korea. As well as changes in visual acuity, IOP, and VF results, changes in various ocular surface symptoms and signs were also statistically analyzed. In addition, post hoc analysis was performed on the statistically significant results. That is, for signs or symptoms that were improved at $90 \mathrm{D}$ after replacement of eyedrops, further analysis was performed to compare the degree of improvement at $45 \mathrm{D}$ and $90 \mathrm{D}$. The other advantage of this paper is that studies on PF latanoprost have not yet been conducted in hospitals other than tertiary hospitals in South Korea. There are differences in the characteristics of glaucoma patients visiting primary and secondary hospitals and tertiary hospitals. That is, patients with stable course of glaucoma usually visit a primary or secondary hospital, and patients with advanced or rapidly progressing glaucoma visit a tertiary hospital. Since there are many glaucoma patients who have been treated at primary and secondary hospitals, our study is meaningful in showing the effectiveness of PF latanoprost in a community-centered medical environment.

The major limitation was that there was no control group that continuously used preserved PGAs. This may degrade the objectivity of evaluating the efficacy of eye drops. Second, due to the nature of the open-label study, both investigators and subjects were already aware of the efficacy of PF latanoprost. This could affect the judgment of the treatment effect and cause information bias. Third, since OSDI and ocular tolerability evaluation are somewhat subjective, the reliability of the study will be improved if objective assessments, such as the Schirmer test or LipiView, are additionally performed. Fourth, the number of study subjects was relatively small, and the study duration was rather short to assess long-term effects. Fifth, BAK-preserved PGAs previously used by patients in this study included latanoprost, bimatoprost and tafluprost. Since there are differences in components and BAK concentrations between eye drops, various factors other than BAK may be involved in improving ocular surface symptoms or signs. However, drug-specific comparison was not performed due to the small sample size. Lastly, since this study was targeted to patients who were already using PGAs, initial IOPs before using eyedrops were often not known. So, baseline IOPs were not included in the analysis. As further study, if comparative study is performed on newly diagnosed patients or after a washout period, it will be possible to analyze the difference in the effect of lowering IOP based on the initial IOP.

In conclusion, the effects of BAK-preserved PGAs and $\mathrm{PF}$ latanoprost on reducing IOP were comparable, but side effects on the ocular surface were much less when PF latanoprost was used. With this efficacy, PF latanoprost can slow the progression of glaucoma by increasing patient compliance and make it possible to treat many glaucoma patients, including glaucoma patients with ocular surface diseases.

\section{Conflict of Interest}

No potential conflict of interest relevant to this article was reported.

\section{References}

1. Tham YC, Li X, Wong TY, et al. Global prevalence of glaucoma and projections of glaucoma burden through 2040: a systematic review and meta-analysis. Ophthalmology 2014;121:2081-90.

2. Kim CS, Seong GJ, Lee NH, et al. Prevalence of primary open-angle glaucoma in central South Korea the Namil study. Ophthalmology 2011;118:1024-30.

3. Kim JH, Kang SY, Kim NR, et al. Prevalence and characteristics of glaucoma among Korean adults. Korean J Ophthalmol 2011;25:110-5.

4. Kim KE, Kim MJ, Park KH, et al. Prevalence, awareness, and risk factors of primary open-angle glaucoma: Korea National Health and Nutrition Examination Survey 20082011. Ophthalmology 2016;123:532-41.

5. Lee AJ, McCluskey P. Clinical utility and differential effects of prostaglandin analogs in the management of raised intraocular pressure and ocular hypertension. Clin Ophthalmol 2010;4:741-64.

6. Tomic M, Kastelan S, Soldo KM, Salopek-Rabatic J. Influence of BAK-preserved prostaglandin analog treatment on the ocular surface health in patients with newly diagnosed primary open-angle glaucoma. Biomed Res Int 2013;2013: 603782.

7. El Hajj Moussa WG, Farhat RG, Nehme JC, et al. Compar- 
ison of efficacy and ocular surface disease index score between bimatoprost, latanoprost, travoprost, and tafluprost in glaucoma patients. J Ophthalmol 2018;2018:1319628.

8. Uusitalo H, Egorov E, Kaarniranta K, et al. Benefits of switching from latanoprost to preservative-free tafluprost eye drops: a meta-analysis of two Phase IIIb clinical trials. Clin Ophthalmol 2016;10:445-54.

9. Choi JA. New classes of glaucoma medical treatment. J Korean Med Assoc 2019;62:497-504.

10. van der Worp E, de Brabander J, Swarbrick HA, Hendrikse F. Evaluation of signs and symptoms in 3- and 9-o'clock staining. Optom Vis Sci 2009;86:260-5.

11. Begley C, Caffery B, Chalmers R, et al. Review and analysis of grading scales for ocular surface staining. Ocul Surf 2019;17:208-20.

12. Cho P, Leung L, Lam A, Choi A. Tear break-up time: clinical procedures and their effects. Ophthalmic Physiol Opt 1998;18:319-24.

13. Charnock C. Are multidose over-the-counter artificial tears adequately preserved? Cornea 2006;25:432-7.

14. Baudouin $\mathrm{C}$, Labbe A, Liang $\mathrm{H}$, et al. Preservatives in eyedrops: the good, the bad and the ugly. Prog Retin Eye Res 2010;29:312-34.

15. Boimer C, Birt CM. Preservative exposure and surgical outcomes in glaucoma patients: the PESO study. J Glaucoma 2013;22:730-5.

16. Misiuk-Hojlo M, Pomorska M, Mulak M, et al. The RE-
LIEF study: tolerability and efficacy of preservative-free latanoprost in the treatment of glaucoma or ocular hypertension. Eur J Ophthalmol 2019;29:210-5.

17. Park J, Lee SJ, Lee KW, Kim JC. Effect of chronic benzalkonium chloride exposure on senescence in trabecular meshwork cells. J Korean Ophthalmol Soc 2019;60:55-61.

18. Garcia-Feijoo J, Munoz-Negrete FJ, Hubatsch DA, Rossi GC. Efficacy and tolerability of benzalkonium chloride-free travoprost in glaucoma patients switched from benzalkonium chloride-preserved latanoprost or bimatoprost. Clin Ophthalmol 2016;10:2085-91.

19. Tokuda N, Kitaoka Y, Matsuzawa A, et al. Changes in ocular surface characteristics after switching from benzalkonium chloride-preserved latanoprost to preservative-free tafluprost or benzalkonium chloride-preserved tafluprost. $J$ Ophthalmol 2017;2017:3540749.

20. Lee SM, Jin SW. Efficacy of preservative-free latanoprost in normal-tension glaucoma with mild to moderate dry eye. J Korean Ophthalmol Soc 2020;61:639-44.

21. Kim YW, Lee SC, Wy S, Bak E. Efficacy and patient tolerability of preservative-free latanoprost in Korean primary open angle glaucoma patients. J Korean Glaucoma Soc 2020;9:10-6.

22. Lee W, Lee $\mathrm{S}$, Bae H, et al. Efficacy and tolerability of preservative-free $0.0015 \%$ tafluprost in glaucoma patients: a prospective crossover study. BMC Ophthalmol 2017;17:61. 